\title{
The Binary Dwarf Carbon Star SDSS J125017.90+252427.6
}

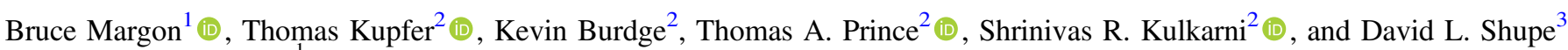 \\ ${ }^{1}$ Department of Astronomy \& Astrophysics, University of California, Santa Cruz, CA 95064, USA \\ ${ }^{2}$ Division of Physics, Mathematics and Astronomy, California Institute of Technology, Pasadena, CA 91125, USA \\ ${ }^{3}$ Infrared Processing and Analysis Center, California Institute of Technology, Pasadena, CA 91125, USA \\ Received 2017 November 22; revised 2018 March 1; accepted 2018 March 2; published 2018 March 19
}

\begin{abstract}
Although dwarf carbon (dC) stars are universally thought to be binaries in order to explain the presence of $C_{2}$ in their spectra while still near main-sequence luminosity, direct observational evidence for their binarity is remarkably scarce. Here, we report the detection of a 2.92 day periodicity in both the photometry and radial velocity of SDSS J125017.90+252427.6, an $r=16.4 \mathrm{dC}$ star. This is the first photometric binary $\mathrm{dC}$, and only the second $\mathrm{dC}$ spectroscopic binary. The relative phase of the photometric period to the spectroscopic observations suggests that the photometric variations are a reflection effect due to heating from an unseen companion. The observed radial velocity amplitude of the $\mathrm{dC}$ component $\left(K=98.8 \pm 10.7 \mathrm{~km} \mathrm{~s}^{-1}\right)$ is consistent with a white dwarf companion, presumably the evolved star that earlier donated the carbon to the $\mathrm{dC}$, although substantial orbital evolution must have occurred. Large synoptic photometric surveys such as the Palomar Transient Factory, which was used for this work, may prove useful for identifying binaries among the shorter-period $\mathrm{dC}$ stars.
\end{abstract}

Key words: binaries: spectroscopic - stars: carbon

\section{Introduction}

Although the first dwarf carbon (dC) star was identified 40 years ago (Dahn et al. 1977), these objects, characterized by prominent $C_{2}$ absorption bands, yet main-sequence luminosity, remain enigmatic in many ways. An excellent review by Green (2013; hereafter "G13") points out that the only plausible source of photospheric $C_{2}$ prior to post-main-sequence evolution is mass transfer from a faster evolving but now inconspicuous binary companion. Aside from the origin of the $C_{2}$ features, $\mathrm{dC}$ stars also pose other interesting problems. The prototype, G77-61, is extraordinarily metal-poor (Plez \& Cohen 2005). Recently, several very-high-velocity $\mathrm{dC}$ stars have also been suggested as candidates for ejecta from binary supernovae (Plant et al. 2016).

Despite wide agreement that $\mathrm{dC}$ stars must be (or have been) binaries, direct evidence for the binary nature of these objects is remarkably scarce. Chiefly thanks to the Sloan Digital Sky Survey (SDSS; York et al. 2000), there are now close to $10^{3} \mathrm{dC}$ stars identified (Margon et al. 2002; Downes et al. 2004; Green 2013; Si et al. 2014), yet only barely more than a handful are directly observed to be binaries. Remarkably, there is only one spectroscopic binary, the prototype dC star G77-61 (Dearborn et al. 1986). About a dozen are known to have $\mathrm{dC} / \mathrm{DA}$ white dwarf composite spectra, and $\sim 20$ show emission lines (G13 and references therein) suggestive of membership in a binary, but perhaps instead due to chromospheric activity on a single star. Only the (one) spectroscopic binary is amenable to even begin quantitative characterization of the system. In this respect, $\mathrm{dC}$ stars frustratingly differ from a variety of other postmass-transfer systems, e.g., barium stars, where essentially all are observed to be binary (McClure et al. 1980). There are very few papers in the literature that report negative results in searches for dC radial velocity variations (cf. Green \& Margon 1994), so it is difficult to judge how extensively this problem has been pursued spectroscopically.

Here, we discuss a new approach to identifying binaries among the $\mathrm{dC}$ stars, and report on the first successful example.

\section{Observations}

\subsection{Photometry}

We utilized the Palomar Transient Factory (PTF; Law et al. 2009; Rau et al. 2009) to search for photometric periodicities in the list of SDSS faint high-latitude carbon stars presented by G13 and Si et al. (2014). PTF data reduction is described in Laher et al. (2014), and the photometric system is discussed in Ofek et al. (2012). The relative photometric calibration uses the prescription of Ofek et al. (2011). Approximately $10^{3}$ objects were examined for periods in the range of $10^{-1}-10^{3}$ days. We used the Vartools Lomb-Scargle algorithm (Hartman \& Bakos 2016) to identify the most probable periods in the light curve.

Although several objects with promising periodicities appeared in this analysis, one object in particular displayed periodic behavior, with very high probability, thus was chosen for further study. The $r=16.4 \mathrm{dC}$ star SDSS J125017.90 +252427.6 (hereafter "J1250") displays a period $P=$ $2.92217 \pm 0.0015$ days, with an $r$-band semi-amplitude of $0.023 \pm 0.002 \mathrm{mag}$, and a formal false occurrence probability, calculated by the Hartman \& Bakos (2016) formalism, of $\sim 10^{-21}$. The uncertainty on the derived period was estimated as described by Kupfer et al. (2013), using a simple Monte Carlo simulation, where $10^{3}$ periodograms were computed, with each iteration deleting a random number of randomly chosen data points, which were then replaced with a duplicate of a different observation. For each such simulated data set, the highest periodogram peak was taken as the orbital period, and the standard deviation of the ensemble of these trial peaks was adopted as the final uncertainty.

In Figure 1 we show a periodogram of an analysis of the $r$-band PTF data on $\mathrm{J} 1250$, consisting of 173 observations obtained with random cadence over an interval of almost 6 years. Figure 2 displays a folded light curve at the best-fit sine wave period, together with residuals from this fit. The phase of the sine wave was fixed, and was obtained from the best-fit radial velocity curve (Section 2.2). Examination of the window 


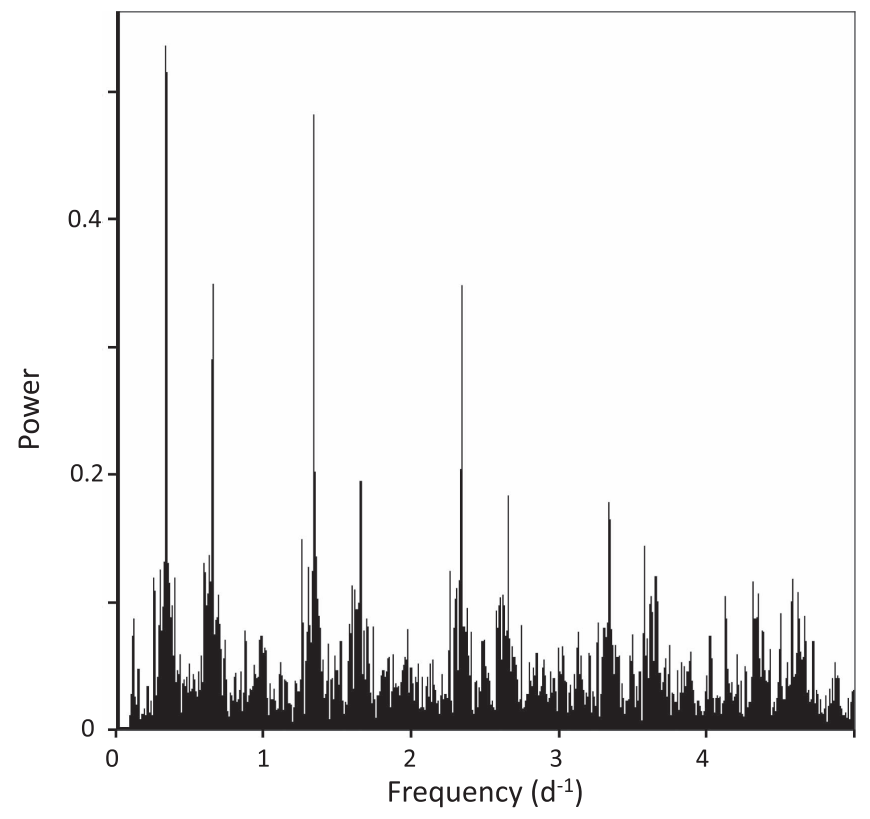

Figure 1. Periodogram of PTF $r$-band photometry of $\mathrm{J} 1250$, derived via the Lomb-Scargle algorithm. The power is an empirical overlap of the periodic signal with a sinusoid at the same frequency, where unity represents perfect overlap. The strong detection of a period at 0.342 days $^{-1}$ and higher harmonics is readily apparent. A second set of peaks at 0.056 days $^{-1}$ is the beat of this primary period with 1 day.

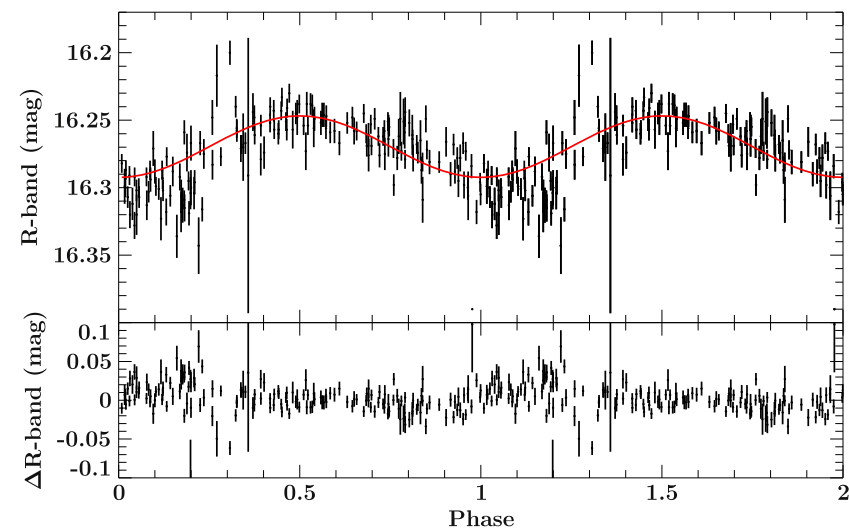

Figure 2. Upper: folded PTF light curve of J1250 at the best-fit period, $P=2.92217$ days, together with a sine fit. For ease of comparison of the photometric and spectroscopic data, the sine wave shown here uses the best-fit photometric period and amplitude, but the best-fit spectroscopic phase. Lower: residuals from the fit.

function (i.e., an identical analysis of an artificial pure sine wave evaluated at the same times as the actual data) reveals that the observed higher harmonics in Figure 1 are not necessarily informative regarding the shape of the light curve, but rather result from a finite-length data set of poorly spaced observations of an underlying period close to an even multiple of one day.

We searched for shorter-term (minutes) photometric variability in J1250 via observations with the Palomar $1.5 \mathrm{~m}$ Telescope on multiple nights in 2017. No significant rapid variations were observed.

There is only one previous mention of J1250 in the literature; G13 first notes it as an SDSS dC star, with a distance estimate of 300 pc. He also remarked on two unusual properties: the star displays strong Balmer emission in the SDSS spectrum (rare, but not unprecedented in the known sample of $\mathrm{dC}$ stars), as well as very weak soft X-ray emission detected by $X M M$ (unique in the G13 sample, but consistent in luminosity with observations of chromospheric/coronal activity in other late type stars).

J1250 appears in the Catalina Real Time Transient Survey (CRTS; Drake et al. 2009), where no significant photometric periodicity is noted. Given the low amplitude of the periodicity that we observe in PTF, this non-detection in CRTS at this magnitude is not surprising.

Only limited other photometric measurements are available in the literature. J1250 has JHK photometry available in 2MASS (Skrutskie et al. 2006). The object is not detected by GALEX (Bianchi et al. 2014).

\subsection{Spectroscopy}

We obtained six spectra of J1250 on 2017 April 20-22, using the Echellette Spectrograph and Imager (ESI) (Sheinis et al. 2002) at the Keck II telescope. This instrument covers the 3900-10000 $\AA$ range, and the 0!'75 slit employed yields a resolution $R=7000$. A typical exposure time was $600 \mathrm{~s}$. We also obtained lower-resolution spectra on three additional nights in 2017 using the Palomar $5 \mathrm{~m}$ reflector and the Double Beam Spectrograph (Oke \& Gunn 1982). The Keck and Palomar spectra are similar to the SDSS discovery spectrum, displaying deep $C_{2}$ Swan bands, strong (resolved) $\mathrm{NaD}$ doublet absorption, the red $\mathrm{CN}$ bands, and prominent Balmer and $\mathrm{Ca}$ $\mathrm{H} \& \mathrm{~K}$ emission. At the substantially higher signal-to-noise and spectral resolution of the Keck data, numerous other cool star absorption features are also clearly detected, including multiple $\mathrm{Ca} \mathrm{I}$ and $\mathrm{Ca}$ II lines. The Keck spectra resolve the Balmer emission; $\mathrm{H} \alpha$ has $0.6 \AA$ FWHM. Comparison of spectra on different nights makes it immediately apparent that there are substantial velocity shifts. The Balmer emission velocities in each spectrum agree to within the measurement errors with the velocities of multiple $\mathrm{Ca}$ I, $\mathrm{Ca}$ II, and $\mathrm{NaD}$ absorption lines, so the emission and absorption appear to come from similar locations. In the subsequent analysis we use multiple strong, sharp Balmer emission lines in the Keck spectra for velocity measures, due to their very high signal-to-noise ratios. The Palomar spectra yield consistent velocities, but are not included in the analysis, as their substantially lower resolution fails to resolve the emission lines.

The PTF photometric period determination has excellent precision due to the long time baseline. We have therefore folded the radial velocities determined from the Keck spectra with the best-fit PTF 2.92 day photometric period, and fit these folded data with a sine wave, allowing the amplitude and phase to remain as free parameters. The folded data and resulting best fit are shown in Figure 3; the sine function is clearly a good fit, with a velocity half-amplitude $K=98.8 \pm 10.7 \mathrm{~km} \mathrm{~s}^{-1}$ and systemic velocity $\gamma=-24.7 \pm 4.8 \mathrm{~km} \mathrm{~s}^{-1}$. Phase zero at $\mathrm{HJD}=2457863.6548 \pm 0.001$ days corresponds to when the $\mathrm{dC}$ is closest to us.

\section{Discussion}

Although there are composite spectrum dC stars known, we believe this to be only the second spectroscopic binary, finally complementing the prototype $\mathrm{dC}$, G77-61. However, the parameters of this system are clearly very different. The observed radial velocity variations and 2.92-day period imply a 


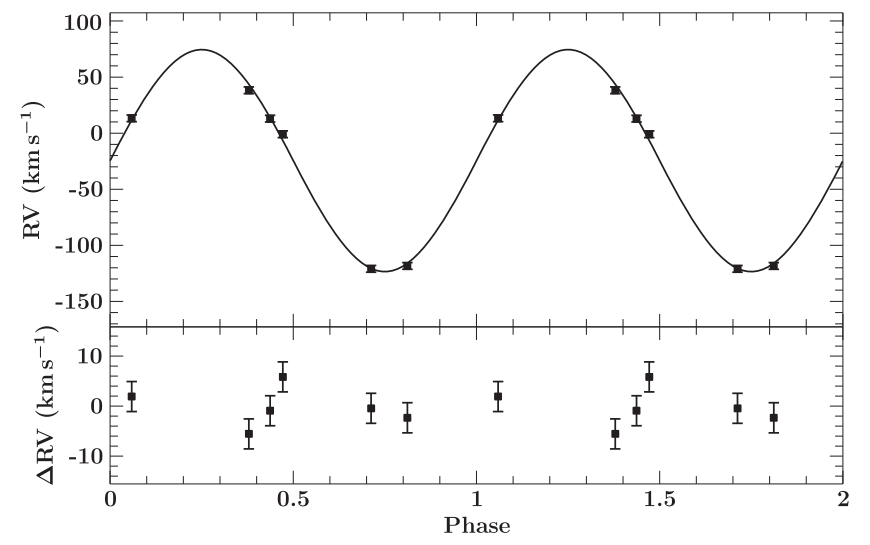

Figure 3. Radial velocity of J1250 vs. orbital phase, from Keck/ESI spectra. The RV data are folded with the photometric orbital period and plotted twice for clarity, with residuals shown below.

mass function $f(M)=0.29 M_{\odot}$. The colors and spectrum of $\mathrm{J} 1250$ (with the exception of the $C_{2}$ bands) suggest that it is a $K$-type $\mathrm{dC}$ (which is the most common). For example, the observed prominent $\mathrm{CN}$ bands and Balmer emission are not found in the rarer, warmer $G$-types (G13), and the latter also fall in a restricted SDSS color wedge that differs from the observed J1250 colors. The mass of the $\mathrm{dC}$ is uncertain, but adopting the $\mathrm{dK}$ assumption, it is likely $\sim 0.3 M_{\odot}$, implying a minimum companion mass $M_{\text {comp }} \sim 0.6 M_{\odot}$. The lack of detectability of the companion in the visible spectrum, plus the requirement that it be highly evolved to have donated the observed $C_{2}$ on the $\mathrm{dC}$, indicates a compact object, and the inferred $M_{\text {comp }} \sim 0.6 M_{\odot}$ is consistent with a typical white dwarf mass. Given the surprisingly short orbital period of 2.92 days, substantial orbital evolution must have occurred.

The existence of radial velocity variations, together with the observed photometric and radial velocity phases, rules out a variety of models for the periodic brightness modulation, such as pulsation or ellipsoidal variations, and suggests that the unseen white dwarf is still sufficiently luminous to heat the face of the $\mathrm{dC}$. The observed Balmer emission might be an additional indicator of the heating, although the data in Table 1 indicate there is no obvious variability of the emission line strength with binary phase. Alternatively, the emission may well be related to stellar activity, as also evidenced by the faint X-ray emission (G13).

\section{Conclusion}

More than 30 years after Dearborn et al. (1986) reported G77-61, the prototype dC star, as a 245-day spectroscopic binary, J1250 finally presents a second example, as well as the first $\mathrm{dC}$ with synchronous photometric variations. The two objects are clearly quite different. The simulations of de Kool \& Green (1995) predicted a bimodal orbital period distribution for $\mathrm{dCs}$, centered on periods of a few years and a few decades. We have considered the possibility that $\mathrm{J} 1250$ is a triple system, with an as yet undiscovered donor star in a wide orbit, as has been suggested for SDSS J1837+40 by G13. However, we know that the observed close companion of J1250 is itself a subluminous, evolved star of the type hypothesized in all dCs. It thus seems unnecessary to invoke an additional, undiscovered subluminous object.

For the sake of completeness, we note that the relationship of $\mathrm{dC}$ stars and the better-studied carbon-enhanced metal-poor
Table 1

ESI Spectra of J1250

\begin{tabular}{lcccc}
\hline \hline $\begin{array}{l}\text { HJD 2457860 } \\
\text { (days) }\end{array}$ & Phase & $\begin{array}{c}\text { Velocity } \\
\left(\mathrm{km} \mathrm{s}^{-1}\right)\end{array}$ & $\begin{array}{c}\mathrm{H}_{\alpha} \mathrm{EW} \\
(\AA)\end{array}$ & $\begin{array}{c}\text { FWHM } \\
(\AA)\end{array}$ \\
\hline 4.763347 & 0.37875 & $38.2 \pm 3.1$ & $5.2 \pm 0.4$ & $0.60 \pm 0.03$ \\
4.932885 & 0.43668 & $12.9 \pm 3.2$ & $5.1 \pm 0.4$ & $0.61 \pm 0.03$ \\
5.034631 & 0.47145 & $-1.0 \pm 3.0$ & $5.4 \pm 0.4$ & $0.68 \pm 0.03$ \\
5.739630 & 0.71234 & $-121.0 \pm 2.9$ & $5.6 \pm 0.4$ & $0.64 \pm 0.03$ \\
6.029278 & 0.81131 & $-118.4 \pm 3.1$ & $5.5 \pm 0.4$ & $0.63 \pm 0.03$ \\
6.753926 & 0.05891 & $13.2 \pm 3.1$ & $5.8 \pm 0.4$ & $0.59 \pm 0.03$ \\
\hline
\end{tabular}

(CEMP) stars (e.g., Aoki et al. 2007) is unclear. Although the latter are mostly giants and subgiants, and they are certainly selected via techniques quite different from $\mathrm{dCs}$, there are a handful with $C_{2}$ bands where model calculations indicate high gravities indicative of dwarfs. Thus, these objects are inferred to be dwarfs via modeling, rather than direct observation of parallaxes or proper motions, as is the case for $\mathrm{dC}$ stars. At least one of these, HE 0024-2523 (Carretta et al. 2002), often referred to as a $\mathrm{CH}$ or even $\mathrm{Pb}$ star, is known to be a spectroscopic binary with $P=3.14$ days and $K=50 \mathrm{~km} \mathrm{~s}^{-1}$ (Lucatello et al. 2003). It may well be the case that the $\mathrm{dC}$ and high-gravity CEMP stars are the precursors of the CEMP giants. An additional related object may be the central star of the planetary nebula G054.2-03.4, which displays Swan bands and has a 1.16 day photometric period (Miszalski et al. 2013).

Because the orbital periods of G77-61 and J1250 are so different, it is quite unclear how many shorter-period $\mathrm{dC}$ systems such as J1250 exist and await discovery via photometric variations, such as those observed here. Current and future large-scale synoptic photometric surveys, coupled with multi-color candidate selection such as that employed by SDSS, may well prove to be a fruitful discovery mechanism.

We are grateful to P. Green for numerous conversations about dC stars, and thank E. Ofek for comments on the manuscript. Observations were obtained with the Samuel Oschin Telescope at the Palomar Observatory as part of the Palomar Transient Factory and Intermediate Palomar Transient Factory project, a scientific collaboration between the California Institute of Technology, Columbia University, Las Cumbres Observatory, the Lawrence Berkeley National Laboratory, the National Energy Research Scientific Computing Center, the University of Oxford, the Weizmann Institute of Science, Los Alamos National Laboratory, the University of Wisconsin, Milwaukee, the Oskar Klein Center, the TANGO Program of the University System of Taiwan, and the Kavli Institute for the Physics and Mathematics of the Universe. Funding for the SDSS and SDSS-II has been provided by the Alfred P. Sloan Foundation, the Participating Institutions, the National Science Foundation, the U.S. Department of Energy, the National Aeronautics and Space Administration, the Japanese Monbukagakusho, the Max Planck Society, and the Higher Education Funding Council for England. The SDSS web site is http://www.sdss.org/. Some of the data presented herein were obtained at the W.M. Keck Observatory, which is operated as a scientific partnership among the California Institute of Technology, the University of California, and the National Aeronautics and Space Administration. The Observatory was made possible by the generous financial support of the W.M. Keck Foundation. The authors wish to 
recognize and acknowledge the very significant cultural role and reverence that the summit of Maunakea has always had within the indigenous Hawaiian community. We are most fortunate to have the opportunity to conduct observations from this mountain.

Facilities: Keck:II (ESI), Sloan, Hale, PO:1.2 m, PO:1.5m.

\section{ORCID iDs}

Bruce Margon (1) https://orcid.org/0000-0002-7837-3363

Thomas Kupfer (1) https://orcid.org/0000-0002-6540-1484

Thomas A. Prince (1) https://orcid.org/0000-0002-8850-3627

Shrinivas R. Kulkarni 10 https://orcid.org/0000-0001-5390-8563

\section{References}

Aoki, W., Beers, T. C., Christlieb, N., et al. 2007, ApJ, 655, 492

Bianchi, L., Conti, A., \& Shiao, B. 2014, AdSpR, 53, 900

Carretta, E., Gratton, R., Cohen, J. G., Beers, T. C., \& Christlieb, N. 2002, AJ, 124,481

Dahn, C. C., Liebert, J., Kron, R. G., Spinrad, H., \& Hintzen, P. M. 1977, ApJ, 216, 757 de Kool, M., \& Green, P. J. 1995, ApJ, 449, 236

Dearborn, D. S. P., Liebert, J., Aaronson, M., et al. 1986, ApJ, 300, 314

Downes, R. A., Margon, B., Anderson, S. F., et al. 2004, AJ, 127, 2838

Drake, A. J., Djorgovski, S. G., Mahabal, A., et al. 2009, ApJ, 696, 870

Green, P. J. 2013, ApJ, 765, 12

Green, P. J., \& Margon, B. 1994, ApJ, 423, 723

Hartman, J. D., \& Bakos, G. Á. 2016, A\&C, 17, 1

Kupfer, T., Groot, P. J., Levita, D., et al. 2013, MNRAS, 432, 2048

Laher, R. R., Surace, J., Grillmair, C. J., et al. 2014, PASP, 126, 674

Law, N. M., Kulkarni, S. R., Dekany, R. G., et al. 2009, PASP, 121, 1395

Lucatello, S., Gratton, R., Cohen, J. G., et al. 2003, AJ, 125, 875

Margon, B., Anderson, S. F., Harris, H. C., et al. 2002, AJ, 124, 1651

McClure, R. D., Fletcher, J. M., \& Nemec, J. M. 1980, ApJL, 238, L35

Miszalski, B., Boffin, H. M. J., \& Corradi, R. L. M. 2013, MNRAS, 428, L39

Ofek, E. O., Frail, D. A., Breslauer, B., et al. 2011, ApJ, 740, 65

Ofek, E. O., Laher, R., Law, N., et al. 2012, PASP, 124, 62

Oke, J. B., \& Gunn, J. E. 1982, PASP, 94, 586

Plant, K. A., Margon, B., Guhathakurta, P., et al. 2016, ApJ, 833, 232

Plez, B., \& Cohen, J. G. 2005, A\&A, 434, 1117

Rau, A., Kulkarni, S. R., Law, N. M., et al. 2009, PASP, 121, 1334

Sheinis, A. I., Bolte, M., Epps, H. W., et al. 2002, PASP, 114, 851

Si, J., Luo, A., Li, Y., et al. 2014, SCPMA, 57, 176

Skrutskie, M. F., Cutri, R. M., Stiening, R., et al. 2006, AJ, 131, 1163

York, D. G., Adelman, J., Anderson, J. E., Jr., et al. 2000, AJ, 120, 1579 